\title{
Staging Treatment of Atrial septal Defect with Severe Pulmonary Arterial Hypertension: Case Report
}

\author{
Feng li*, Liang Fu, Xian Jin, Jinlong Zhao, Yinkai Ni, Zhexin Lu and Zonghui Chen \\ Department of Cardiovascular Surgery, Shanghai Jiao Tong University, China
}

*Corresponding author: Feng li, Department of Cardiovascular Surgery, Shanghai Jiao Tong University Affiliated Sixth People's Hospital, 600 Yishan Rd, Shanghai 200233, People's Republic of China, China

\begin{abstract}
Atrial septal defect is the most common type of congenital heart diseases. Interventional closure is currently the best treatment for atrial septal defect. However, atrial septal defect combined with severe pulmonary hypertension remains a clinical problem. We applied a perforated occlude to the clinical treatment of atrial septal defect with severe pulmonary hypertension and achieved good results. As the pulmonary pressure gradually went down, the reserved hole of the occlude can be blocked latterly
\end{abstract}

Keywords: Manual fenestrated device closure; Atrial septal defect; Pulmonary hypertension

\section{Introduction}

Closure of an atrial septal defect (ASD) with severe pulmonary artery hypertension (PAH) is discouraged, since a serious rise in pulmonary pressure will be fatal. In patients with ASD and severe pulmonary hypertension, it is critically important to identify whether the PHT is reversible before considering transcatheter or surgical closure. As to the reversible PHT, manual fenestrated device closure will be effective. Here we reported a patient with ASD and severe PHT who firstly received percutaneous closure of the defect with a manual fenestrated device.

\section{Case Report}

A 26 years old female patient, with no known cardiovascular history, was admitted to hospital for a heart murmur at the second intercostal space on the left sternal border. She can do light to moderate physical activity without developing dyspnea. The transthoracic echocardiography indicated atrial septal defect (Type II, diameter 25mm); left-to-right bidirectional shunt, severe pulmonary hypertension (PASP: $114 \mathrm{mmHg}$ ); right atrium and ventricular dilatation with tricuspid severe regurgitation (Figure 1). Blood gas analysis showed: $\mathrm{PH}: 7.36, \mathrm{PaO}_{2}: 77.2 \mathrm{mmHg}, \mathrm{PaCO}_{2}$ : $36.1 \mathrm{mmHg}$. The electrocardiogram was normal. Chest radiograph demonstrated pulmonary artery segment bulging suggesting sever
PTH. Right heart catheter verified sever PTH (pulmonary artery systolic pressure was $112 \mathrm{mmHg}$ ). Therefore, repair or closure of the ASD would be a high risk for a probable surge of pulmonary artery pressure post the procedure. After serious consideration, we decided to treat the girl by a manual fenestrated closure device. Under general anesthesia and transesophageal echocardiography (TEE) guidance, the patient was inserting a handmade fenestrated closure device (reserved hole $5 \mathrm{~mm}$, Figure 2) through the right femoral vein, occlusion of the occluder in advance. After the device was released, TEE showed a slight left-to-right shunt. After the procedure, the patient's oxygen saturation was $100 \%$. On the second day post procedure, the girl was discharged. Echocardiography was performed at the 2 nd month and 6th month post operation, and the PASP was $68 \mathrm{mmhg}$ and $34 \mathrm{mmhg}$ respectively. Meanwhile, tricuspid regurgitation was reduced to mild reflux. Right atrium and right ventricle size also decreased when compared with preoperative. Therefore, the patient's PHT was reversible since the pulmonary artery pressure decreased significantly after the procedure. Thus, after careful evaluation, we take the second stage of procedure to re-plug the $5 \mathrm{~mm}$ reserved hole in the ASD closure device to eliminate the shunt (see Figure 3). The operation was successful, and the patient was discharged the next day. 


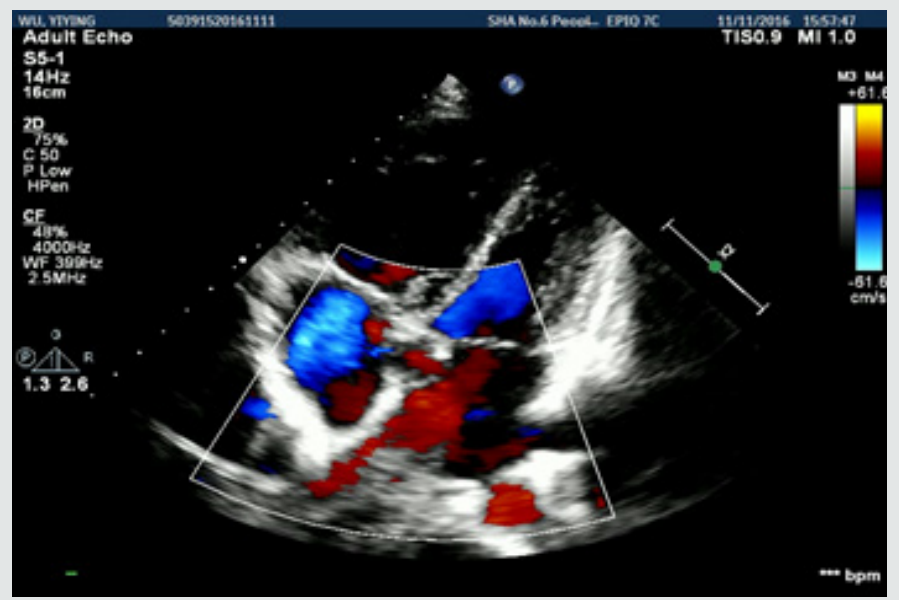

Figure 1: Transthoracic echocardiography suggested secondary atrial septal defect.

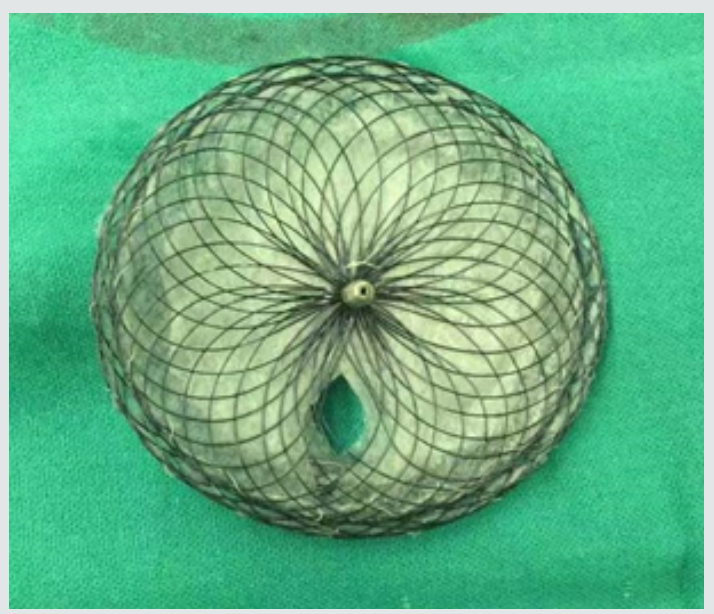

Figure 2: Occlusion of the occluder in advance reserved hole $5 \mathrm{~mm}$.

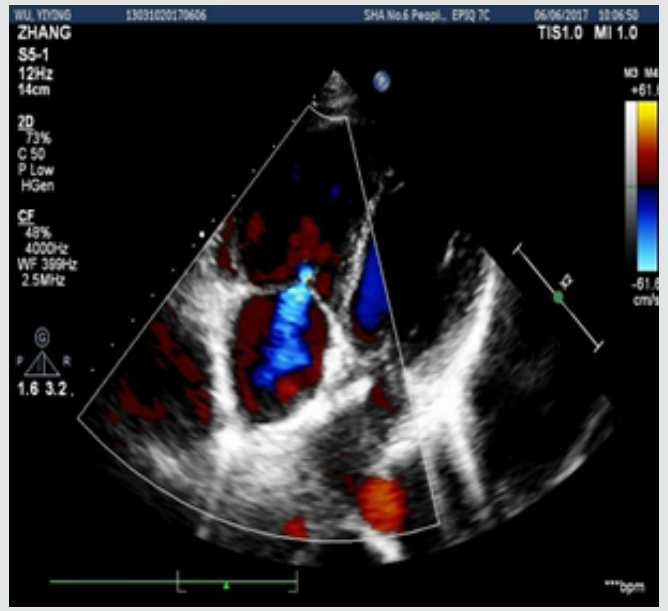

Figure 3: Re-plugged the $5 \mathrm{~mm}$ reserved hole.

\section{Discussion}

Atrial septal defect is the most common congenital heart disease. Although considered to be a benign disease, it can still cause serious complications and fatalities with pathophysiological changes [1-2]. Interventional sealing has become the most effective way to treat secondary atrial septal defects. However, for atrial septal defect with severe pulmonary hypertension, whether the operation under direct vision or percutaneous closure is high risky. In this 
case, we performed two stage procedure to treat the ASD. Firstly, we closed the ASD with a handmade fenestrated closure device. Such procedure has been proved effective because the occlusion can reduce interatrial shunt, meanwhile the handmade reserved small hole will prevent the surge of postoperative pulmonary hypertension [3]. Postoperative follow-up also confirmed that pulmonary artery pressure decreased significantly, tricuspid regurgitation also reduced, and the heart was remodeled. When the patient's pulmonary arterial pressure reduced to a safe range, we completely blocked the reserved hole through the femoral venous esophageal ultrasound guidance.

In summary, the occluder with reserved holes has a good effect on the treatment of patients with secondary atrial septal defect with severe pulmonary hypertension. According to the follow-up results after surgery, the reserved holes can be re-plugged if necessary. However, large samples of clinical data are still needed for further study and validation.

\section{References}

1. Campbell M (1970) Natural history of atrial septal defect.J Cardiovascular Clinics 32(6): 820.

2. Schuldt A, Assmann T (1968) Natural history and prognosis of atrial septal defect. J Circulation 37(5): 805-815.

3. Bruch L, Winkelmann A, Sonntag S (2010) Fenestrated occluders for treatment of ASD in elderly patients with pulmonary hypertension and/ or right heart failure. Journal of Interventional Cardiology 21(1): 44-49. (c) This work is licensed under Creative

To Submit Your Article Click Here: Submit Article

DOI: $10.32474 /$ SCSOAJ.2019.03.000172

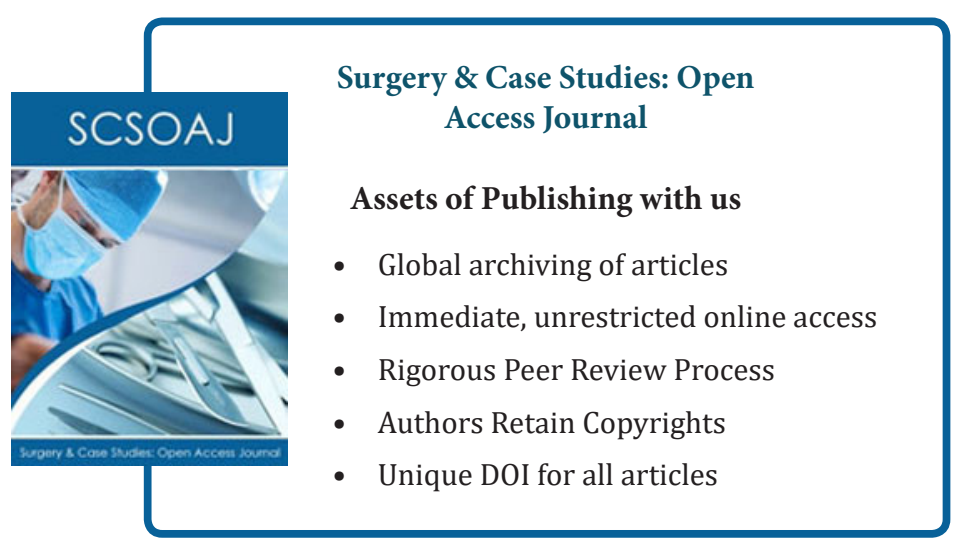

